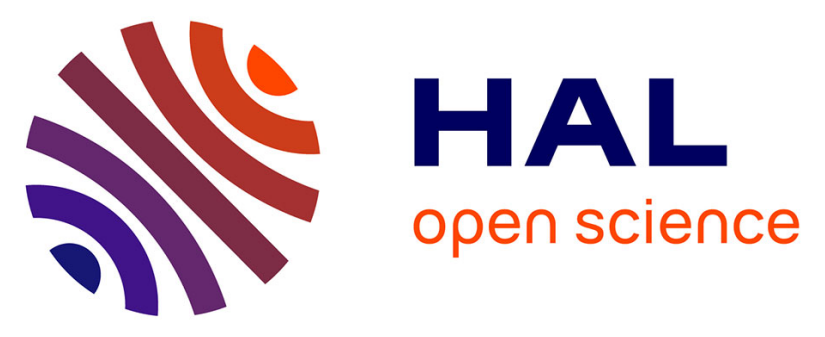

\title{
Molecular determinants of substrate and inhibitor specificities of the Penicillium griseofulvum family 11 xylanases.
}

Marine Cervera Tison, Gwenaëlle André-Leroux, Michaël Lafond, Jacques

Georis, Nathalie Juge, Jean-Guy Berrin

\section{To cite this version:}

Marine Cervera Tison, Gwenaëlle André-Leroux, Michaël Lafond, Jacques Georis, Nathalie Juge, et al.. Molecular determinants of substrate and inhibitor specificities of the Penicillium griseofulvum family 11 xylanases.. Biochimica et Biophysica Acta Proteins and Proteomics, 2009, 1794 (3), pp.438-45. 10.1016/j.bbapap.2008.11.024 . hal-02668875

\section{HAL Id: hal-02668875 \\ https://hal.inrae.fr/hal-02668875}

Submitted on 31 May 2020

HAL is a multi-disciplinary open access archive for the deposit and dissemination of scientific research documents, whether they are published or not. The documents may come from teaching and research institutions in France or abroad, or from public or private research centers.
L'archive ouverte pluridisciplinaire HAL, est destinée au dépôt et à la diffusion de documents scientifiques de niveau recherche, publiés ou non, émanant des établissements d'enseignement et de recherche français ou étrangers, des laboratoires publics ou privés.

\section{()(1)(2)}

Distributed under a Creative Commons Attribution - ShareAlikel 4.0 International 


\title{
Molecular determinants of substrate and inhibitor specificities of the Penicillium griseofulvum family 11 xylanases
}

\author{
Marine Cervera Tison a , Gwénaëlle André-Leroux ${ }^{\mathrm{b}}$, Mickaël Lafond ${ }^{\mathrm{a}}$, Jacques Georis ${ }^{\mathrm{c}}$, Nathalie Juge ${ }^{\mathrm{d}, *}$, \\ Jean-Guy Berrin ${ }^{\mathrm{a}, 1}$ \\ a Biosciences ISM² UMR-CNRS-6263, Université Paul Cézanne Aix Marseille III, Av. Escadrille Normandie-Niemen, 13397 Marseille Cedex 20, France \\ b Institut Pasteur, Unité de Biochimie Structurale, 25 rue du Dr Roux, 75724 Paris Cedex XV, France \\ ${ }^{c}$ Puratos Group, rue Bourrie 12, 5300 Andenne, Belgium \\ d Institute of Food Research, Colney Lane, Norwich NR4 7UA, UK
}

\section{A R T I C L E I N F O}

\section{Article history:}

Received 4 July 2008

Received in revised form 21 November 2008

Accepted 24 November 2008

Available online 13 December 2008

\section{Keywords:}

Glycoside hydrolase family 11

Wheat arabinoxylan

Xylo-oligosaccharide

Site-directed mutagenesis

Xylanase inhibitor

Homology modeling

\begin{abstract}
A B S T R A C T
Penicillium griseofulvum possesses two endo-(1,4)- $\beta$-xylanase genes, PgXynA and PgXynB, belonging to family 11 glycoside hydrolases. The enzymes share $69 \%$ identity, a similar hydrolysis profile i.e. the predominant production of xylobiose and xylotriose as end products from wheat arabinoxylan and a specificity region of six potential xylose subsites, but differ in terms of catalytic efficiency which can be explained by subtle structural differences in the positioning of xylohexaose in the PgXynB model. Site-directed mutagenesis of the "thumb" region revealed structural basis of PgXynB substrate and inhibitor specificities. We produced variants displaying increased catalytic efficiency towards wheat arabinoxylan and xylo-oligosaccharides and identified specific determinants in PgXynB "thumb" region responsible for resistance to the wheat xylanase inhibitor XIP-I. Based on kinetic analysis and homology modeling, we suggested that Pro130 ${ }_{\text {PgXynB }}$, Lys131 $1_{\text {PgXynB }}$ and Lys132 $2_{\text {PgXnn }}$ hamper flexibility of the loop forming the "thumb" and interfere by steric hindrance with the inhibitor.
\end{abstract}

(c) 2008 Elsevier B.V. All rights reserved.

\section{Introduction}

$\beta$-1,4-Xylan, the most abundant component of hemicelluloses in the cell walls of monocots and hard woods, is a heteropolysaccharide consisting of a backbone of $\beta$-1,4-linked D-xylopyranosyl residues substituted with L-arabinosyl, 4-O-methyl-glucuronosyl, and acetyl side chains [1,2]. As xylan varies in structure between different plant species, complete hydrolysis requires a large variety of cooperatively acting enzymes such as xylanases, xylosidases, arabinofuranosidases, glucuronidases, acetylxylan esterases, ferulic acid esterases, and $p$ coumaric acid esterases [3-5]. All of these enzymes act cooperatively to convert xylan into its constituent sugars. Of these, endo-(1,4)- $\beta$ xylanases (xylanases; EC 3.2.1.8) are of particular significance since

Abbreviations: A/X, arabinose to xylose ratio; DNS, Dinitrosalicylic acid; GH, Glycoside hydrolase; GST, glutathione-S-transferase; HPAEC-PAD, High Performance Anion Exchange Chromatography-Pulsed Amperometric Detection; LVAX, Low viscosity wheat arabinoxylan; MALDI-MS, Matrix-Assisted Laser Desorption Ionization/Mass Spectrometry; PfXynC, xylanase C from Penicillium funiculosum; PgXynA, xylanase A from Penicillium griseofulvum; PgXynB, xylanase B from Penicillium griseofulvum; TAXI, Triticum aestivum xylanase inhibitor; TLXI, thaumatin-like xylanase inhibitor; XIP-I, xylanase inhibitor protein I; X1, xylose; X2, xylobiose; X3, xylotriose; X4, xylotetraose; $\mathrm{X} 5$, xylopentose; X6, xylohexaose

* Corresponding author. Fax: +44(0)1603 255038.

E-mail address: nathalie.juge@bbsrc.ac.uk (N. Juge).

1 Present address: UMR 1163 INRA de Biotechnologie des Champignons Filamenteux, ESIL, 163 Avenue de Luminy, Case Postale 925, 13288 Marseille Cedex 09, France. they can hydrolyse the internal 1,4- $\beta$-D-xylosidic linkages within the xylan backbone to produce short-chain xylo-oligosaccharides of varying length [5]. Many xylanolytic microorganisms secrete multiple isoforms of xylanases that can cooperate to enhance the hydrolysis of the complex xylan [6], fungi being the most potent producers $[7,8]$. Xylanolytic enzymes of microbial origin have received great attention due to their biotechnological utility and potential application in a range of industrial processes but the observed effects vary depending on xylanase specificities [9]. In the past few years, the potential applications of xylanases for bioconversion of lignocellulosic feedstocks to fuel-grade ethanol have been of particular interest to researchers.

Based on amino acid sequence similarities, xylanases are mainly clustered into families 10 and 11 of glycoside hydrolases (GH) (www. cazy.org [10]). The two families have different molecular structures, molecular weights, and catalytic properties (see [5] for a review). Family 11 consists of 20-30 kDa molecular weight proteins with 21 structures folded as a $\beta$-jelly roll available from both bacteria and fungi. GH10 xylanases exhibit higher affinity for shorter linear $\beta-1,4-$ xylo-oligosaccharides than GH11 xylanases [11]. GH10 xylanases have a $(\beta / \alpha)_{8}$-barrel as a catalytic domain and typically contain one or more carbohydrate-binding domains, which increase the effective concentration of the catalytic domain on polymeric substrates [12]. The structure of GH11 xylanases has been described as a partially closed right hand [13]. It consists of a single domain folding into two $\beta$ - 
sheets, which are packed against each other, and one $\alpha$-helix. The two $\beta$-sheets are strongly twisted and form a cleft on one side of the protein in which the active site is situated. This cleft is covered by a long loop region, called the "thumb" region and partly closed on one side by the cord, a long irregular loop with a well-defined structure. A consequence of the difference in structure is their difference in substrate specificity. The active site of GH10 xylanases is a shallow groove, which is reflected in their specificity towards a lower number of unsubstituted consecutive xylose units. In contrast, GH11 xylanases show higher affinity towards a larger number of unsubstituted consecutive xylose units because of their cleft-shaped active site [11]. Multiple xylanases also occur within each category of GH families 10 and 11 [6]. Typically many introns are present in GH10 xylanase genes from filamentous fungi [14] whereas GH11 xylanase genes contain one or two introns [15].

Microbial GH10 and GH11 xylanases are target of proteinaceous xylanase inhibitors present in cereals, Triticum aestivum xylanase inhibitor (TAXI), xylanase inhibitor protein (XIP), and thaumatin-like xylanase inhibitor (TLXI) (for a review, see [16]). The specificity of these inhibitors is dependent on the source and nature of the xylanase tested: TAXI and TLXI inhibitors can inhibit GH11 xylanases from both fungal and bacterial origin but not those of GH10 whereas XIP-type inhibitors typically inhibit GH10 and GH11 endoxylanases from fungal sources (for a review, see [9]). Several lines of evidence point towards a role of these inhibitor proteins in plant defense: (i) their lack of inhibition activity against endogenous xylanases (ii) their ability to inhibit xylanases from pathogens Fusarium graminearum and Botrytis cinerea (iii) the fact that some TAXI and XIP genes are wound and pathogen inducible and (iv) the homologies of TLXI and XIP with pathogenesis-related (PR) proteins of family 5 (PR-5) and 8 (PR-8), respectively [16].

The Penicillia are mostly saprophytic in nature and numerous species have been exploited in the production of xylanolytic enzymes (reviewed in [17]). The presence of family 10 and family 11 xylanases has been reported for Penicillium purpurogenum [18], Penicillium citrinum [15,19], Penicillium funiculosum [20,21] and Penicillium capsulatum [22,23]. A xylanase from Penicillium griseofulvum (PgXynA) was isolated using a screening method based on the ability to digest wheat flour arabinoxylan and the corresponding gene was cloned and expressed in Aspergillus oryzae [24]. We have previously characterised PgXynA heterologously expressed in Escherichia coli [25]. Computational design methods was applied to guide structure-based sitedirected mutagenesis analyses of PgXynA enzymes with changed specificity, altered $\mathrm{pH}$ profile, and improved overall catalytic activity [26]. In the present study, we report the production and enzymatic, biochemical and structural characterisation of $\operatorname{PgXynB}$, a second xylanase from P. griseofulvum with 69\% identity to PgXynA [24]. The enzyme was characterised in terms of kinetics towards arabinoxylan, xylo-oligosaccharides products formation and inhibition sensitivity. We engineered PgXynB variants displaying increased catalytic efficiency towards wheat arabinoxylan and xylo-oligosaccharides and identified unique PgXynB determinants in the "thumb" region responsible for resistance to the wheat xylanase inhibitor XIP-I. The PgXynA and PgXynB xylanases constitute an ideal system for the study of structure-function relationships within family 11 xylanases.

\section{Materials and methods}

\subsection{Materials, plasmids, and strains}

E. coli DH5 $\alpha$ strain was used for DNA manipulation and BL21 strain (DE3) pLys for protein expression (Novagen, Merck Biosciences, Fontenay, France). High purity salt-free oligonucleotides, pDONrZeo and pDEST17 plasmids were from Invitrogen (Cergy Pontoise, France), restriction endonucleases and DNA modifying enzymes were from Promega (Madison, WI, USA), pGEX-1 $\lambda$ T expression vector, GST glutathione agarose beads, and thrombin protease from Amersham Biosciences (GE healthcare, Orsay, France), BugBuster and benzonase from Novagen, lysosyme, $\beta$-D-xylose, and dinitrosalicylic acid from Sigma (Lyon, France), and low viscosity wheat arabinoxylan (LVAX) and 1,4- $\beta$-D-xylo-oligosaccharides (xylose X1, xylobiose X2, xylotriose $\mathrm{X} 3$, xylotetraose X4, xylopentose X5, and xylohexaose X6) from Megazyme International (Wicklow, Ireland). Purified wheat arabinoxylan substrates with different arabinose to xylose ratio $(A / X)$ were kindly provided by C. Courtin (Katholieke University Leuven, Belgium).

\subsection{PgXynB cloning and site-directed mutagenesis}

The intron present in $\operatorname{PgXynB}$ genomic sequence [27] was eliminated by overlap extension method [28] using primers hybridizing with each exon of PgXynB. Two independent PCR reactions (PCRI and PCRII) were performed using PgXynB as template (10 ng), together with $1.25 \mathrm{U}$ of DNA polymerase (Prime STAR DNA polymerase TAKARA) and $0.2 \mathrm{mM}$ dNTP. The primers used in this study are given in Table 1. In a first PCR run, PCRI and PCRII reactions were carried out using PGB1F and PGB2R primers (20 pmol) for PCRI and PGB3F and PGB4R primers (20 pmol) for PCRII in $50 \mu \mathrm{L}$ volume and for 25 cycles of denaturation $\left(1 \min 98^{\circ} \mathrm{C}\right)$, annealing $\left(2 \min\right.$ at $48^{\circ} \mathrm{C}$ ) and extension $\left(1.5 \mathrm{~min}\right.$ at $\left.72{ }^{\circ} \mathrm{C}\right)$ in a Mastercycler ${ }^{\circledR}$ gradient thermocycler (Eppendorf, Hambourg, Germany). The resulting PCRI (195 bp) and PCRII (426 bp) products were gel-purified using the QIAquick gel extraction kit (Qiagen, Courtaboeuf, France). The final PCR run was carried out with $150 \mathrm{ng}$ of purified PCRI and PCRII products, $1.25 \mathrm{U}$ of DNA polymerase and $0.2 \mathrm{mM}$ dNTP. After five cycles of denaturation $\left(1 \mathrm{~min}\right.$ at $\left.94{ }^{\circ} \mathrm{C}\right)$, annealing $\left(2 \mathrm{~min}\right.$ at $42{ }^{\circ} \mathrm{C}$ ) and extension $(3 \mathrm{~min}$ at $72{ }^{\circ} \mathrm{C}$ ), either PGBHIF and PGBEIR or PGBGWF and PGBGWR primers (20 pmol) were added and the reaction was subjected to 25 cycles of denaturation $\left(1 \mathrm{~min}\right.$ at $\left.94{ }^{\circ} \mathrm{C}\right)$, annealing $\left(2 \mathrm{~min}\right.$ at $53{ }^{\circ} \mathrm{C}$ ) and extension $\left(1.5 \mathrm{~min}\right.$ at $\left.72{ }^{\circ} \mathrm{C}\right)$.

The final PCR product (597 bp) obtained with PGBHIF and PGBEIR primers was gel-purified, digested by BamHI and EcoRI, and ligated into the pGEX-1 $\lambda \mathrm{T}$ plasmid. After transformation in E. coli $\mathrm{DH} 5 \alpha$ strain, the recombinant vector pGEX-1 $\lambda \mathrm{T} / \operatorname{PgXyn} B$ was checked by restriction mapping and double stranded DNA sequencing (Genome Express, Meylan, France).

The final PCR product ( $627 \mathrm{bp}$ ) amplified with the help of the att tagged primer pair, PGBGWF and PGBGWR, was combined with the donor vector (pDONrZeo) following manufacturer's instructions (Gateway ${ }^{\circledR}$ Cloning, Invitrogen) resulting in the formation of an entry clone (pDONrZeo/PgXynB). After sequencing, the PgXynB coding sequence was further transferred by a recombination reaction from the Gateway ${ }^{\circledR}$ entry clone to the pDEST17 destination vector, allowing expression of $\operatorname{PgXynB}$ with a poly-histidine tagged (His) $)_{6}$ at the Nterminal to produce (His) ${ }_{6} \mathrm{PgXynB}$.

Table 1

Oligonucleotide sequences used for elimination of the intron sequence and construction of pGEX1 $\lambda \mathrm{T} / \operatorname{PgXynB}$ and pDEST17/PgXynB

\begin{tabular}{ll}
\hline Name & Oligonucleotide sequences $\left(5^{\prime} \rightarrow 3^{\prime}\right)$ \\
\hline$P G B 1 F$ & GCT CTC TTT ACA AGC CAA ACT GGA G \\
$P G B 2 R$ & ATA GTG GAT GTT CCG GGC ACT ACC AGT GCT CCA GCC CTT GCC \\
$P G B 3 F$ & GGT AGT GCC CGG AAC ATC CAC TAT GCT GGA GAT TTC AAA CCC \\
$P G B 4 R$ & TAA CCA AAC AGA CAT GTC AGC GTA TCC \\
$P G B H I F$ & TTT TTT GGA TCC GCT CTC TTT ACA AGC CAA ACT GGA G \\
$P G B E I R$ & TTT TTT GAA TTC TCA CCA AAC AGA CAT GTC AGC GTA TCC \\
$P G B G W F$ & GGG ACA AGT TTG TAC AAA AAA GCA GGC TTA GCT CTC TTT ACA AGC \\
& CAA ACT GGA G \\
PGBGWR & GGG CCA CTT TGT ACA AGA AAG CTG GGT TTA CCA AAC AGA CAT GTG \\
\hline
\end{tabular}

$\overline{\text { Restriction sites and att-flanked sites are shown in bold and bold underlined, }}$ respectively. 
$\mathbf{a}$
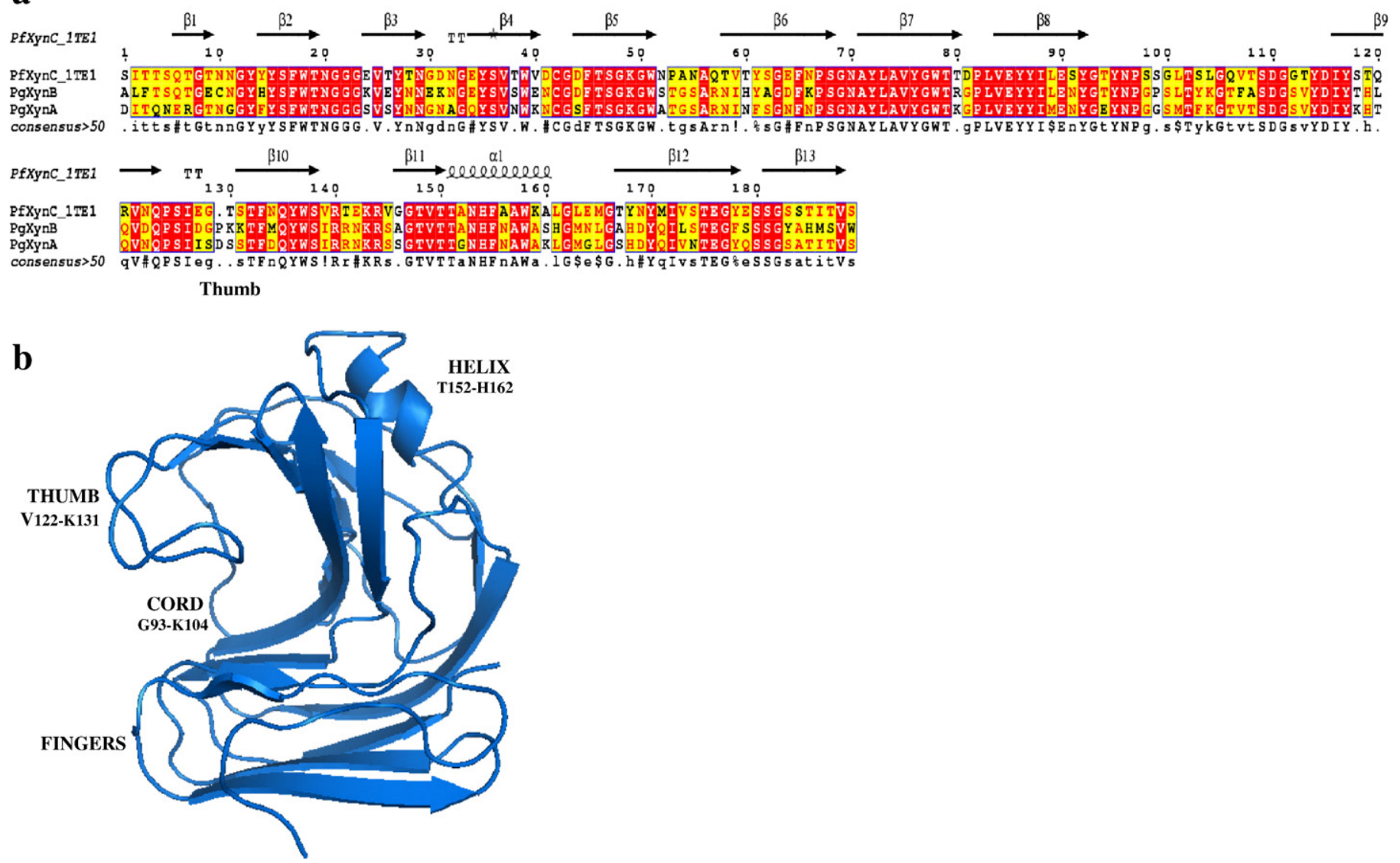

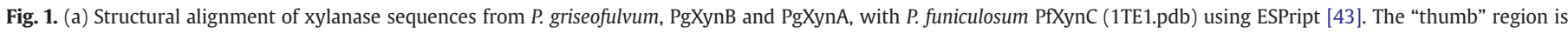

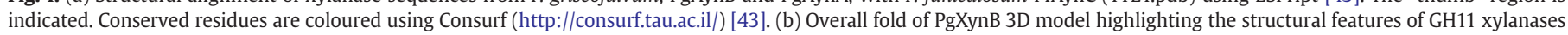
("thumb", "palm", "fingers" and "cord") as described in [13]. The ribbon diagram representation was drawn using the pymol software (www.pymol.org/funding.html).

Mutations were introduced into the pGEX-1入T/PgXynB plasmid using the QuickChange ${ }^{\circledR}$ XL site-directed mutagenesis kit (Stratagene) following manufacturer's recommendations using a pair of overlapping complementary oligonucleotides for each mutation designed to contain the corresponding nucleotide changes.

\subsection{PgXynB expression and purification of recombinant enzyme}

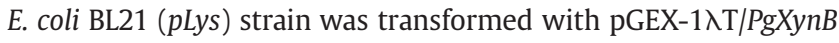
and pDEST17/PgXynB. Recombinant clones were grown at $37{ }^{\circ} \mathrm{C}$ in LB media with shaking at $200 \mathrm{rpm}$. Expression of GST/PgXynB was performed as described in [25]. Expression of (His) 6 PgXynB was induced with $0.05 \% \mathrm{~L}$-arabinose (w/v, Sigma) when OD600 $=0.6$. After incubation at $37{ }^{\circ} \mathrm{C}$ for $4 \mathrm{~h}$, the cells were centrifuged at $8500 \mathrm{rpm}$ for $30 \mathrm{~min}$ at $4{ }^{\circ} \mathrm{C}$ and stored at $-80^{\circ} \mathrm{C}$ until use.

Bacterial lysis, purification and cleavage of GST/PgXynB wild-type and mutants were carried out as previously described [25]. Recombinant (His) ${ }_{6} P g X y n B$ was purified by affinity chromatography following manufacturer's instructions (His-Bind kit, Novagen). Briefly, after bacterial lysis, the supernatant was loaded onto a Ni-NTA column and the recombinant protein was eluted with $400 \mathrm{mM}$ imidazole in sodium phosphate buffer $\mathrm{pH}$ 8. Fractions were assayed for xylanase activity, pooled and dialysed against Mcllvaine's buffer $\mathrm{pH} 4.5$.

\subsection{Protein assays, electrophoresis and mass spectrometry analysis}

The protein concentration was determined using the Bio-Rad protein assay kit with bovine serum albumin as the standard. SDSPAGE was performed in $12 \%(\mathrm{w} / \mathrm{v})$ polyacrylamide gel (Bio-Rad,
Marnes-la-Coquette, France) using a Pharmacia LMW electrophoresis calibration kit. Native IEF was carried out at $4{ }^{\circ} \mathrm{C}$ in the Bio-Rad gel system, using ampholine carriers of pH range 3.0-6.0 (Sigma) and pI standards ranging from 4.45 to 8.2 (Bio-Rad). Proteins were visualized either by Coomassie or IEF gel staining solution (Bio-Rad). MALDI mass spectra were obtained on an Ettan Maldi-Tof Pro (GE Healthcare Uppsala, Sweden) operating in positive linear mode with delayed extraction. The samples were treated as previously described [25].

\subsection{Xylanase activity and inhibition assays}

Purified xylanase activity was measured using the method described in [29]. Briefly, $20 \mu \mathrm{L}$ of enzyme (PgXynB) was mixed with $180 \mu \mathrm{L}$ of substrate (LVAX or A/X with different ratio) in Mcllvaine's buffer $\mathrm{pH} 4.5$ at $30{ }^{\circ} \mathrm{C}$ for $5 \mathrm{~min}$. The reaction was terminated by the addition of $300 \mu \mathrm{L}$ dinitrosalicylic acid (DNS) reagent, $1 \%(\mathrm{w} / \mathrm{v})$, and boiled for $5 \mathrm{~min}$. The reactions were cooled and centrifuged for $5 \mathrm{~min}$ at $13000 \mathrm{rpm}$ and $200 \mu \mathrm{L}$ was transferred to a microtitre plate. The absorbance at $550 \mathrm{~nm}$ was measured relative to a xylose standard curve $\left(0-180 \mu \mathrm{g} / \mathrm{mL}^{-1}\right)$. One unit of xylanase activity was defined as the amount of protein that released $1 \mu \mathrm{mol}$ of xylose $/ \mathrm{min}$ at $30^{\circ} \mathrm{C}$ and $\mathrm{pH}$ 4.5. Optimal $\mathrm{pH}$ for xylanase activity was estimated using LVAX $\left(10 \mathrm{mg} / \mathrm{mL}^{-1}\right)$ in Mcllvaine's buffer in a $\mathrm{pH}$ range of 2.6 to 7.6. Optimal temperature was estimated at temperatures ranging from 5 to $50{ }^{\circ} \mathrm{C}$. For determination of apparent Michaelis-Menten constants, the initial velocities of the enzymes were measured at $30{ }^{\circ} \mathrm{C}$ in Mcllvaine's buffer, $\mathrm{pH} 4.5$, with substrate concentrations ranging from 3 to $30 \mathrm{mg} /$ $\mathrm{mL}$. The kinetic parameters were estimated using weighted nonlinear squares regression analysis with the Grafit software (Biosoft, 
Table 2

Kinetic parameters of PgXynB and PgXynA wild-type xylanases and PgXynB mutants on wheat arabinoxylan (LVAX)

\begin{tabular}{lclll}
\hline & $\begin{array}{l}K_{\mathrm{m}(\mathrm{app})} \\
\left(\mathrm{mg} \mathrm{mL}^{-1}\right)\end{array}$ & $k_{\text {cat }}\left(\mathrm{s}^{-1}\right)$ & $\begin{array}{l}k_{\text {cat }} / K_{\mathrm{m}(\mathrm{app})} \\
\left(\mathrm{mL} \mathrm{s}^{-1} \mathrm{mg}^{-1}\right)\end{array}$ & $K_{\mathrm{i}}(\mathrm{nM})$ \\
\hline wt-PgXynB & $44.7 \pm 3.3$ & $1166 \pm 59$ & 26.1 & n.d. \\
$\Delta \mathrm{P} 130$ PgXynB & $41.4 \pm 7.0$ & $1104 \pm 126$ & 24.0 & n.d. \\
K131S/K132S PgXynB & $39.4 \pm 2.4$ & $1592 \pm 65$ & 40.4 & n.d. \\
$\Delta \mathrm{P} 130 / K 131 S / K 132 S$ PgXynB & $33.8 \pm 6.3$ & $1537 \pm 184$ & 45.5 & 16.5 \\
wt-PgXynA $^{\mathrm{a}}$ & $3.8 \pm 0.7$ & $545 \pm 29$ & 143 & n.d. \\
\hline
\end{tabular}

n.d. not detectable (up to 1:30 enzyme:inhibitor molar ratio).

a Data from [25].

Cambridge, UK). Inhibition assays of the Penicillium xylanases were determined using LVAX at $30{ }^{\circ} \mathrm{C}$ and $\mathrm{pH}$ 4.5. Recombinant PgXynA, PgXynB and PfXynC [25] were incubated with XIP-I [30] up to an inhibitor/enzyme molar ratio of 30:1.

\subsection{High Performance Anion Exchange Chromatography-Pulsed Amperometric Detection (HPAEC-PAD)}

Xylo-oligosaccharides and their products generated after xylanase hydrolysis were analysed by HPAEC (Dionex, Sunnyvale, CA, USA) equipped with a CarboPac PA-100 analytical column $(250 \times 4 \mathrm{~mm})$, a GP40 gradient pump, and a AS3500 auto sampler. The hydrolysis was carried out using appropriate enzyme and substrate concentrations in Mcllvaine's buffer $\mathrm{pH}$ 4.5. The enzymatic reaction was stopped by the

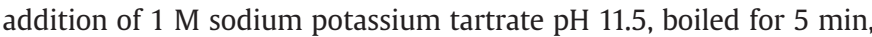
cooled down and centrifuged for $5 \mathrm{~min}$ at $13000 \mathrm{rpm}$, prior to injection $(20 \mu \mathrm{L})$ on the HPAEC system. Elution $\left(1 \mathrm{~mL} / \mathrm{min}^{-1}\right)$ was carried out using a 20 -min linear gradient program from $100 \% \mathrm{~A}$ ( $80 \mathrm{mM} \mathrm{NaOH}$ ) to $60 \% \mathrm{~A}$ and $40 \% \mathrm{~B}$ (NaOAC $500 \mathrm{mM}$; NaOH $80 \mathrm{mM}$ ). The effluent was monitored using an ED40 electrochemical detector. Calibration curves were constructed using xylo-oligosaccharides (X1 to X6) from which response factors were calculated (PeakNet program, Dionex) and used to estimate the amount of product released in test incubations. To evaluate the activity of $\operatorname{PgXynB}$ (wildtype and mutants) on xylo-oligosaccharides, initial slopes of progress curves were used to determine the catalytic efficiency $\left(k_{\text {cat }} / K_{\mathrm{m}}\right)$ of the reaction following the equation of Matsui [31]. All assays were carried out in duplicate.

\subsection{Homology modeling and docking of xylohexaose}

The xylanase of P. funiculosum (PfXynC; 1TE1) [32] was selected as the closest structure to align with PgXynB using Wu_Blast alignment analysis (http://www.ebi.ac.uk/Tools/). The alignment of the two sequences using ClustalW2 from the ebi [33] confirmed PfXynC as 3D template for PgXynB. Homology modeling was performed with Modeler 9v3 [34], based on the coordinates of the chain B of the xylanase of PfXynC. Thirty models of PgXynB were generated that satisfy the spatial restraints issued from the alignment with the target protein. Two models were selected with respect to the score function that derives from a Modeler analysis and to the correct stereochemistry that was validated by PROCHECK [35]. The models were minimized with Charmm, implemented in Accelrys ${ }^{\odot}$ and one PgXynB model was selected according to its potential energy value. For the model of the structure complexed to a xylohexaose, the ligand was positioned in the catalytic groove of $\operatorname{PgXynB}$ with respect to its positioning in the highly homologous PgXynA. The docking was optimized through a cascade of minimizations and short molecular dynamics with Charmm, as previously described for PgXynA [26]. The three mutations $\Delta \mathrm{P} 130 / \mathrm{K} 131 \mathrm{~S} / \mathrm{K} 132 \mathrm{~S}$ were introduced in the sequence of PgXynB wild type and aligned with the sequence of PfXynC. Similarly, the triple mutant $\triangle \mathrm{P} 130 / \mathrm{K} 131 \mathrm{~S} / \mathrm{K} 132 \mathrm{~S}$ was homology modeled based on the chain B of PfXynC as the template structure and refined with the identical protocol of minimization used for PgXynB wild type.

\section{Results and discussion}

\subsection{Functional expression, characterisation and molecular modeling of $\operatorname{PgXynB}$}

PgXynB was produced in E. coli BL21 cells as a fusion with GST (glutathione-S-transferase) or a poly-histidine (His) 6 tag, at the $\mathrm{N}$ terminus end. Optimum expression yields were obtained at $17{ }^{\circ} \mathrm{C}$ after 18-h induction for GST/PgXynB and at $37{ }^{\circ} \mathrm{C}$ after 5 -h induction for (His) ${ }_{6} P g X y n B$. After purification, a major band of $21 \mathrm{kDa}$ and $23 \mathrm{kDa}$ was seen on SDS-PAGE corresponding to purified PgXynB (after proteolysis of GST/PgXynB by thrombin) and purified (His) ${ }_{6} \operatorname{PgXynB}$, respectively (not shown). The yields of purified PgXynB and (His) ${ }_{6} \operatorname{PgXynB}$ were estimated to be around $3 \mathrm{mg} / \mathrm{L}$ of culture. Although both purified xylanases were functionally expressed displaying activity towards LVAX, the specific activity of thrombin-cleaved PgXynB was 5-fold higher than (His) $)_{6} \operatorname{PgXynB}$. Since this may be due to the presence of the poly-histidine tagged sequence at the $\mathrm{N}$-terminus end of the recombinant xylanase, the thrombin-cleaved recombinant enzyme expressed using the GST expression system was used in the rest of the study.

Mass spectrometry confirmed the presence of single component of $21300 \mathrm{Da}$ in excellent agreement with the calculated masses of 21300.2 Da. IEF revealed one single isoform of pI 6.8, in agreement with the theoretical pI. The effects of $\mathrm{pH}$ on the activity of PgXynB revealed over $50 \%$ activity in the $\mathrm{pH}$ range of 3.5 to 5.5 with an optimum $\mathrm{pH}$ of 4.5 . Recombinant PgXynB was stable at $30^{\circ} \mathrm{C}, \mathrm{pH} 4.5$ for 240 min, with more than $85 \%$ of the activity remaining (not shown).

Since no crystal structure of $P$. griseofulvum xylanases is available, a molecular model of PgXynB was performed using the P. funiculosum xylanase PfXynC as the 3D template (1TE1) (82\% similarity and 69\% strict identity with PgXynB) (Fig. 1a and b). PgXynB exhibited the overall $\beta$ jelly-roll shape typical of GH11 xylanases, reminiscent of a right hand with the fingers formed by the two $\beta$-sheets packed against each other, the palm by the twisted part of one $\beta$-sheet and the $\alpha$-helix (between Thr152 $2_{\text {PgXynB }}$ and His $\left.162_{\mathrm{PgXynB}}\right)$, the cord by a loop positioned between Gly93 $3_{\operatorname{PgXyn}}$ and Lys104 $4_{\operatorname{PgXyn}}$ and the "thumb" by a hairpin loop between Val122 $2_{\text {PgXynB }}$ and Lys131 $1_{\text {PgXynB }}$ (Fig. 1b). The cleft corresponding to the active site is lined with aromatic residues Tyr76 $6_{\text {ggXynB }}$, Tyr87 ${ }_{\operatorname{PgXyn}}$, Trp138 ${ }_{\text {PgXynB }}$ and Tyr171 $1_{\text {ggXynB }}$ strictly similar to PgXynA and the two catalytic residues, Glu85 ${ }_{\text {PgXynB }}$ (catalytic nucleophile) and Glu177 $7_{\text {PgXynB }}$ (catalytic acid-base) are positioned at $6.7 \AA$ distance (carbonyl oxygen distance), entirely consistent with the catalytic apparatus of a "retaining" glycoside hydrolase. The superimposition of the PgXynB model with that of PfXynC 3D structure led to the root mean square deviations of $0.2 \AA$. The significant structural differences between PfXynC and PgXynB are located between strand $\beta 5$ and $\beta 6$ (Ala52 $2_{\text {PgXynB }}-$ Ser $55_{\text {PgXynB }}$ ), on the cord (Gly93 ${ }_{\text {PgXynB }}-$ Lys $104_{\text {PgXynB }}$ ) and mainly within the thumb region (Gln121 $1_{\text {ggXynB }}$-Lys 132 $2_{\text {ggXynB }}$ ) that includes the insertion of Pro130 ${ }_{\text {PgXynB }}$ with the two flanking lysine residues Lys $131_{\operatorname{PgXynB}}$ and Lys132 $2_{\operatorname{PgXynB}}$ that are not conserved in PgXynA (Fig. 1a).

Table 3

Specific activities of PgXynB and PgXynA wild-type xylanases and PgXynB mutants on wheat arabinoxylan fractions with different arabinose to xylose ratio $(\mathrm{A} / \mathrm{X})$

\begin{tabular}{lrrr}
\hline & \multicolumn{3}{l}{ Specific activity $\left(\mathrm{U} \mathrm{mg}^{-1}\right)$} \\
\cline { 2 - 4 } & $\mathrm{A} / \mathrm{X} 0.36$ & $\mathrm{~A} / \mathrm{X} \mathrm{0.48}$ & $\mathrm{A} / \mathrm{X} \mathrm{0.69}$ \\
\hline wt-PgXynB & $721 \pm 33$ & $393 \pm 13$ & $176 \pm 22$ \\
$\Delta$ P130 PgXynB & $584 \pm 29$ & $298 \pm 20$ & $148 \pm 11$ \\
K131S/K132S PgXynB & $862 \pm 65$ & $440 \pm 27$ & $212 \pm 38$ \\
$\Delta$ P130/K131S/K132S PgXynB & $1245 \pm 93$ & $702 \pm 30$ & $311 \pm 19$ \\
wt-PgXynA & $1365 \pm 64$ & $1159 \pm 47$ & $909 \pm 54$ \\
\hline
\end{tabular}




\subsection{Enzyme kinetics and substrate docking}

The kinetic parameters of PgXynB xylanase were determined at $\mathrm{pH}$ 4.5 and $30{ }^{\circ} \mathrm{C}$ using LVAX as substrate. PgXynB hydrolysed LVAX with a rate of $1166 \mathrm{~s}^{-1}$ and an apparent affinity of $44.7 \mathrm{mg} \mathrm{mL}^{-1}$ (Table 2). The catalytic efficiency of PgXynB was 5.5-fold lower than PgXynA, mostly

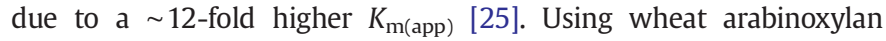
fractions of varying $\mathrm{A} / \mathrm{X}$ ratio, wt- $\mathrm{PgXynB}$ showed highest activity on low substituted xylan: A/X 0.36>A/X 0.49>A/X 0.68 (Table 3). In order to evaluate the mode of action of PgXynB, the products generated against highly polymeric substrate and on short substrates ranging from xylotetraose to xylohexaose were analysed by HPAEC-PAD. PgXynB displayed typical endo-activity against wheat arabinoxylan (LVAX) i.e. a mixture of xylo-oligosaccharides was generated during the initial stages of hydrolysis (Fig. 2). As the reaction continued, the oligosaccharides were progressively degraded yielding principally xylobiose and xylotriose towards the end of the reaction (Fig. 2). This characteristic is common to PgXynA and PfXynC GH11 xylanases that yield xylobiose and xylotriose as main end reaction products [25]. PgXynB had no detectable activity on xylobiose and xylotriose and low activity on xylotetraose but cleaved xylopentaose and xylohexaose rapidly (Fig. 3). PgXynB exhibited an endo-mode of action against xylo-oligosaccharides, as shown on polymeric substrate. For example, xylohexaose was hydrolysed to xylotriose, xylopentaose to xylobiose and xylotriose; whereas xylotetraose was cleaved, initially, to produce mainly xylobiose and small amount of xylotriose and xylose (Fig. 3). Initial rate data from hydrolysis of individual oligomers at $30{ }^{\circ} \mathrm{C}$ and $\mathrm{pH} 4.5$ indicated that the catalytic efficiency $\left(k_{\text {cat }} / K_{\mathrm{m}}\right)$ increased with increasing chain length $(\mathrm{n})$ of oligomer up to $n=6$ (Table 4 ). The relative $k_{\text {cat }} / K_{\mathrm{m}}$ of $\mathrm{PgXynB}$ on xylotetraose, xylopentaose, and xylohexaose was 1:12:129. Noteworthy, the catalytic efficiency value for PgXynB on xylopentaose $\left(7.4 \times 10^{4} \mathrm{~min}^{-1}\right.$ $\left.\mathrm{M}^{-1}\right)$ was 6-fold lower than that for PgXynA $\left(4.6 \times 10^{5} \mathrm{~min}^{-1} \mathrm{M}^{-1}\right)$ (Table 4). The relatively high activity of $\operatorname{PgXynB}$ against xylohexaose and the production of xylotriose from this substrate suggest that its specificity region spans at least six xylose units.

In order to obtain structural insights for the substrate binding differences between PgXynA and PgXynB, xylohexaose was modeled in the binding cleft of PgXynB. As for PgXynA [26], xylohexaose covered subsites $(-3)$ to $(+3)$ (Fig. 4a and b), in accordance with the kinetic analyses reporting predominant production of xylotriose
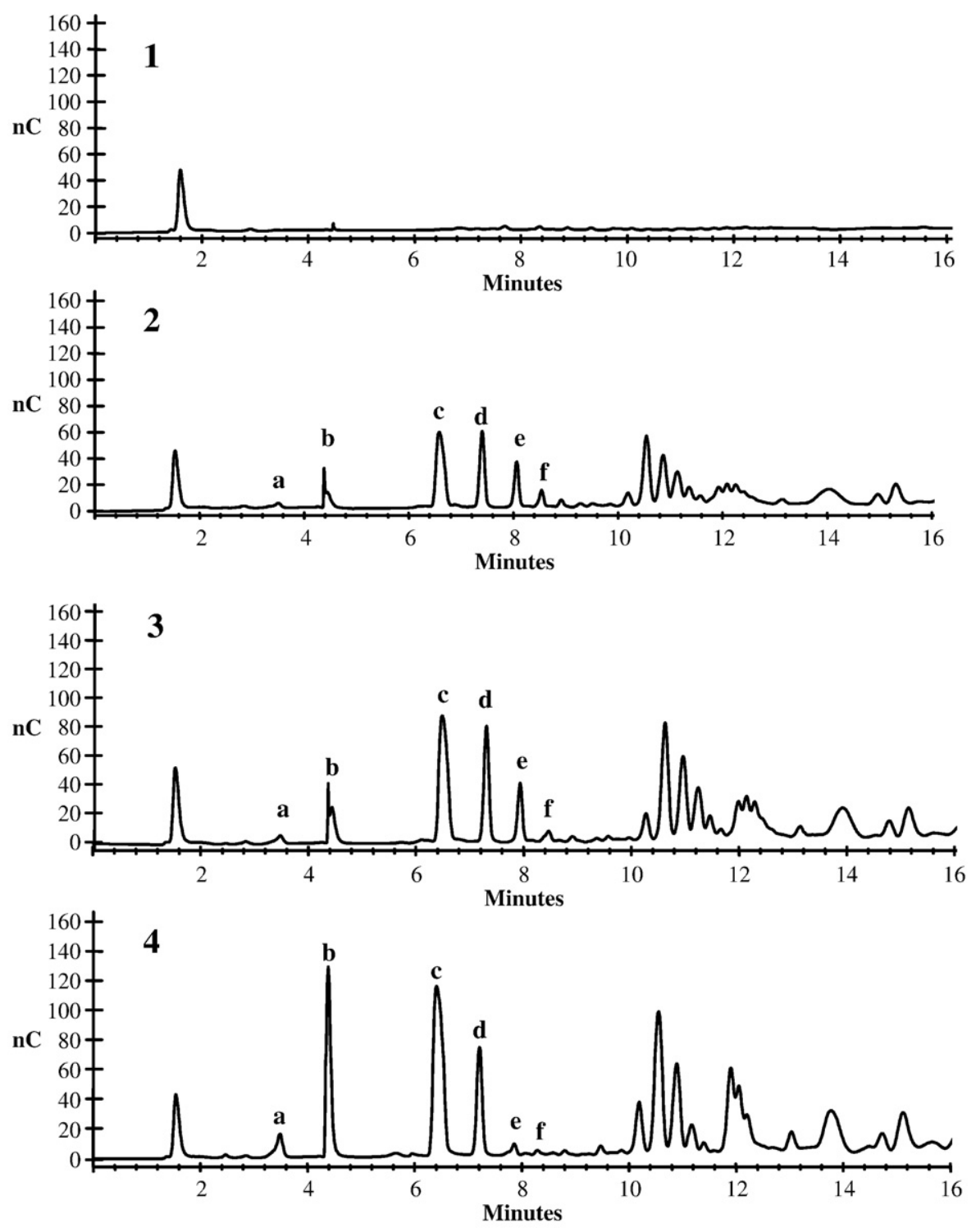

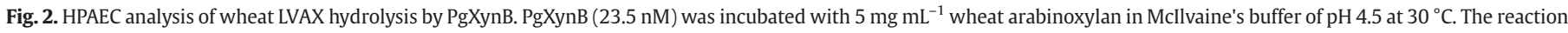

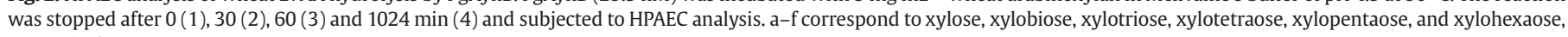
respectively. 

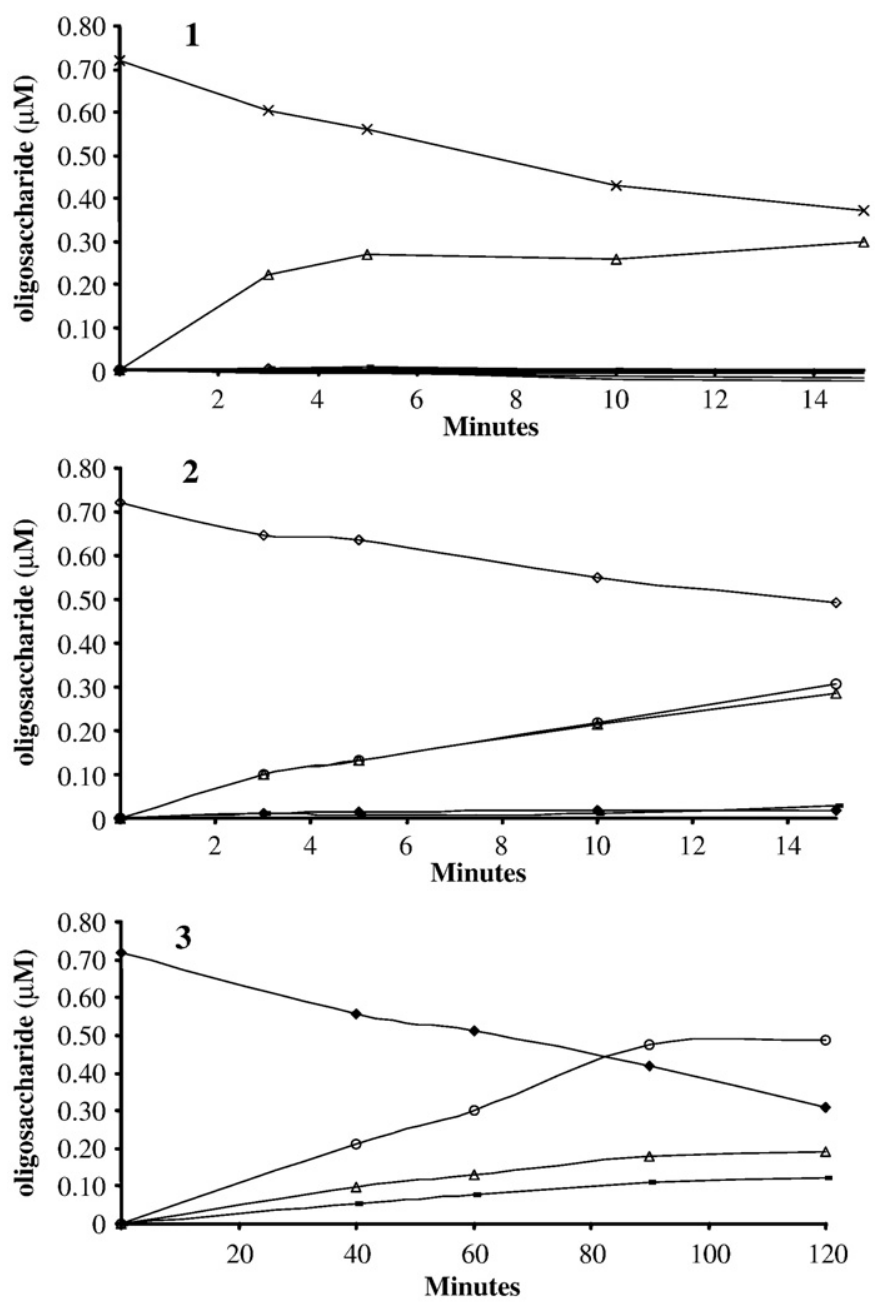

Fig. 3. Progress curve of the xylo-oligosaccharides generated by $\operatorname{PgXynB}$ after hydrolysis of xylohexaose (1), xylopentaose (2), and xylotetraose (3). Recombinant enzymes were incubated with $20 \mu \mathrm{M}$ of xylo-oligosaccharides in Mcllvaine's buffer pH 5.5 at $30^{\circ} \mathrm{C}$. The quantity of xylose $(-)$, xylobiose $(O)$, xylotriose $(\Delta)$, xylotetraose $(\bullet)$, xylopentaose $(\diamond)$, and xylohexaose $(x)$ produced during the course of the reaction is indicated. The concentrations of enzymes used were $14.1 \mathrm{nM}(1), 141 \mathrm{nM}(2)$, and $392 \mathrm{nM}$ (3). Values represent the mean of two independent experiments.

from xylohexaose (Fig. 3). The residues involved in the binding of xylohexaose in PgXynB and PgXynA, either through hydrogen bonding or stacking are shown in Fig. 4. These include conserved residues defining analogous subsites, namely Ile127 $7_{\operatorname{PgXynB}}$ in subsite -3 , Trp18PgXynB and Pro125 $5_{\text {ggXynB }}$ in subsite -2 , Tyr76 ${ }_{\text {PgXynB }}$ and $G \ln 121_{\text {PgXynB }}$ in subsite -1 , Tyr95 ${ }_{\text {PgXynB }}$ and Trp138 $8_{\text {PgXynB }}$ in subsite +2 and Ser $100_{\text {PgXynB }}$ in subsite +3 . The xylose moiety at subsite -1 demonstrated a skew/boat distortion which can be considered as the precursor geometry of the transition state conformation observed by X-ray diffraction for the oligosaccharide bound to B. agaradhaerens xylanase [36]. This is in agreement with the recently solved structure of the family 11 "environmental" xylanase EnXyn11A in complex with a decorated xylotriose showing a boat distortion of the sugar at the -1 subsite [37]. A detailed comparison of PgXynA and PgXynB 3D models showed a significant shift (up to $2 \AA$ on the aglycone side) of the xylohexaose positioned in the active cleft of the xylanases, mainly through differences in the positioning of both cord and thumb loops (Fig. 4). In the PgXynB model, the thumb residues Pro125 $5_{\text {ggXnnB }}$, Gly129 $9_{\text {PgXynB }}$ and Ile127 $7_{\text {PgXynB }}$ are shown to be involved in subsites -3 and -2 , respectively whereas the cord residues Tyr95 ${ }_{\text {ggXynB }}$, Pro97 ${ }_{\text {PgXynB }}$ and Gly98 ${ }_{\text {PgXynB }}$ which homologous residues in PgXynA define subsite +2 are involved with Pro99PgXynB in the binding of xylose at subsite +3 in PgXynB. In addition, several sequence variation between PgXynA and PgXynB could contribute to the difference in binding specificity: $A s n 5_{\operatorname{PgXynA}}$ is replaced by Ser $5_{\text {PgXynB }}$ which side chain would be too short to hydrogen bond the sugar; Asp130 $0_{\text {PgXynA }}$ is replaced by Pro130 $0_{\mathrm{PgXynB}}$ which affects flexibility

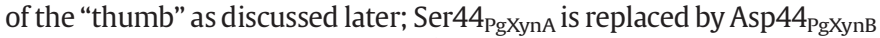
that displaces Trp18 $8_{\text {ggXynB }}$ over $2 \AA$ away from its original position in PgXynA, thus affecting substrate binding; and Tyr179 $9_{\text {PgXynA }}$ is replaced by Phe179 $9_{\text {PgXynB }}$ which lacks an hydroxyl group to hydrogen bond the substrate and which aromatic ring is likely to be located too far to form van der Waals interactions with the substrate (Fig. 4). Together, those divergences could explain the difference in the energy of interaction (36 kcal mol ${ }^{-1}$ ) between PgXynA and PgXynB, probably due to PgXynA forming a more stable complex with xylohexaose than PgXynB. The energy of interaction between the oligosaccharide and its protein receptor was calculated as the sum of electrostatic (columbic) and van der Waals interaction energies using the Lennard-Jones 6-12 potential functions [38]. This difference is fully consistent with the lower catalytic efficiency of PgXynB (5.5-fold) compared to PgXynA, due to a $\sim 12$-fold higher $K_{\mathrm{m}(\mathrm{app})}$.

\subsection{Inhibition sensitivity and molecular engineering of the "thumb"}

The wheat protein inhibitor XIP-I is generally known to inhibit fungal but not bacterial GH11 xylanases. The structural basis for the inhibition of family 11 xylanases by XIP-I was provided by the detailed analysis of the 3D structure of XIP-I in complex with PfXynC [32]. Inhibition is mediated by the insertion of a XIP-I П-shaped loop (148 XIP-I $\left.153_{\text {XIP-I }}\right)$ into the enzyme active site but interactions with regions (thumb and palm) surrounding the entrance of the active site groove are also important recognition determinants for XIP-I, with the overall shape and amino acid composition of the thumb playing a significant role in inhibitor binding [26,32,37,39-41]. As also reported for PgXynA [25] and in contrast to most other fungal GH11 xylanases tested [42], PgXynB showed no reduced xylanase activity up to a XIP-I/PgXynB ratio of 30:1. The resistance of $P$. griseofulvum xylanases to XIP-I could be explained by the structural determinants of XIP-I specificity around the "thumb" region of the enzyme. From the 3D model and sequence alignments, we postulated that the insertion of a Proline at position 130 in PgXynB (which is absent in the XIP-I-sensitive PfXynC) and/or the replacement of the following Serines by Lysines $\left({ }^{129} \mathrm{SDSS}^{132}{ }_{\mathrm{PgXynA}} \rightarrow{ }^{129} \mathrm{GPKK}^{132}{ }_{\text {PgXynB }}\right.$ ) or the combination of both could be responsible for the resistance of PgXynB to XIP-I. Mutations $\Delta$ P130, K131S/K132S and $\Delta$ P130/K131S/ K132S were introduced by site-directed mutagenesis. All mutant and wild-type xylanases were expressed in E. coli, purified as previously described and characterised in terms of molecular mass using mass spectrometry, pI and activity. The specific activity and kinetics parameters $\left(k_{\mathrm{cat}}, K_{\mathrm{m}(\mathrm{app})}, K_{\mathrm{i}}\right)$ of PgXynB mutants were determined using LVAX as substrate (Table 2). The mutants retained optimum $\mathrm{pH}$ comparable to the wt-PgXynB (not shown). A slight pI shift was observed for K131S/K132S and $\Delta \mathrm{P} 130 / \mathrm{K} 131 \mathrm{~S} / \mathrm{K} 132 \mathrm{~S}$ mutants that displayed a pI of 6.5 whereas the $\mathrm{pI}$ of $\triangle \mathrm{P} 130$ remained similar to the wild-type, i.e. 6.8. In order to evaluate the mode of action of the K131S/K132S and $\Delta \mathrm{P} 130 /$ K131S/K132S mutants, HPAEC-PAD was used to analyse the hydrolysis products generated on highly polymeric substrate, wheat arabinoxylan

Table 4

Catalytic efficiency of PgXynA and PgXynB wild-type xylanases and PgXynB mutants on xylo-oligosaccharides

\begin{tabular}{lllll}
\hline & \multicolumn{3}{l}{$k_{\text {cat }} / K_{\mathrm{m}}\left(\mathrm{min}^{-1} \mathrm{M}^{-1}\right)$} \\
\cline { 2 - 5 } & \multicolumn{1}{l}{ X6 } & X5 & X4 & X3 \\
\hline wt-PgXynB & $8.0 \times 10^{5}$ & $7.4 \times 10^{4}$ & $6.2 \times 10^{3}$ & N.D. \\
K131S/K132S PgXynB & $1.5 \times 10^{6}$ & $1.9 \times 10^{5}$ & $1.5 \times 10^{4}$ & N.D. \\
AP130/K131S/K132S PgXynB & $1.2 \times 10^{6}$ & $1.8 \times 10^{5}$ & $1.1 \times 10^{4}$ & N.D. \\
wt-PgXynA $^{\text {a }}$ & $2.55 \times 10^{6}$ & $4.6 \times 10^{5}$ & $3.0 \times 10^{4}$ & $1.5 \times 10^{2}$ \\
\hline
\end{tabular}

N.D. Not Determined.

a Data from [25]. 
a

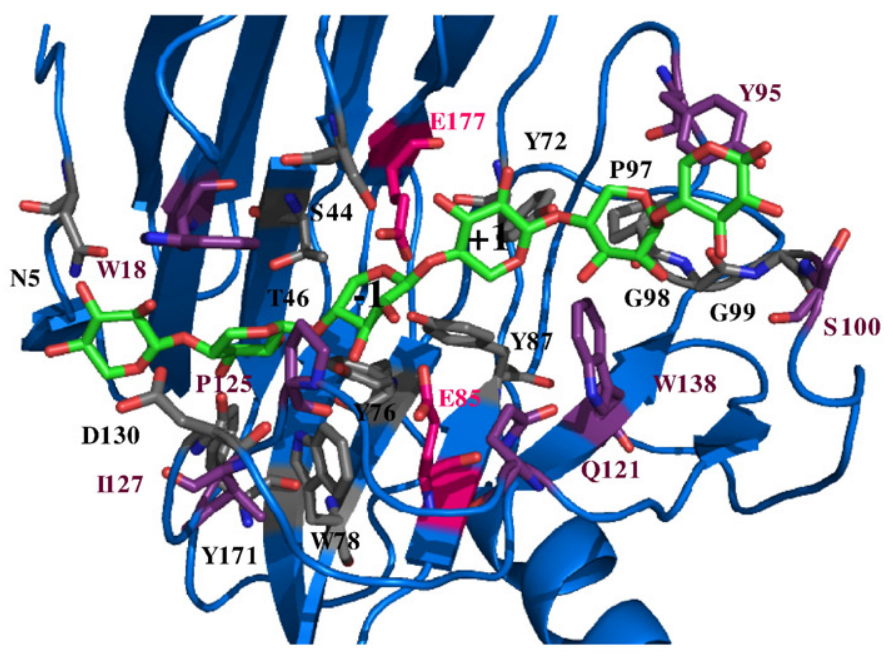

PgXynA b

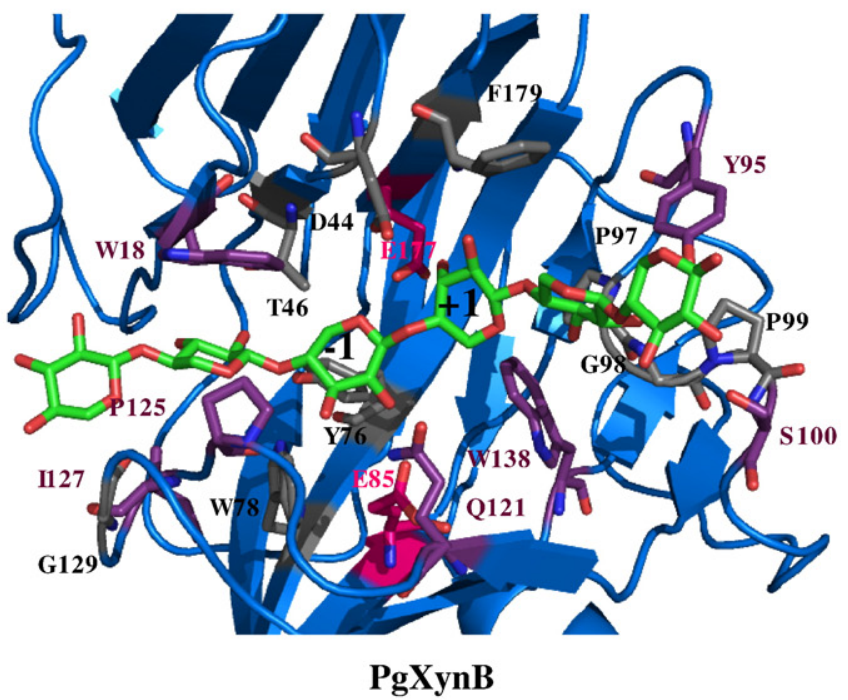

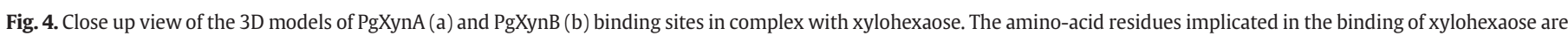

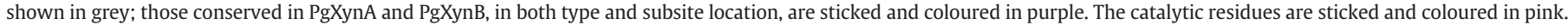

fractions of different arabinose/xylose ratio and oligosaccharides. Although, the mutations did not alter the hydrolysis product profile of LVAX (chromatogram identical to that obtained with the wild-type enzyme, see Fig. 2), K131S/K132S and $\Delta \mathrm{P} 130 / \mathrm{K} 131 \mathrm{~S} / \mathrm{K} 132 \mathrm{~S}$ showed a marked increase in catalytic efficiency due to a $36 \%$ and $31 \%$ increase in turn-over compared to wt-PgXynB, respectively (Table 2). The mutants were also more active on wheat arabinoxylan fractions with varying $\mathrm{A} / \mathrm{X}$ ratio, with a maximum increase of up to $\sim 50 \%$ on $\mathrm{A} / \mathrm{X} 0.36$ compared to wt-PgXynB (Table 3). The increase in catalytic efficiency of K131S/K132S and $\triangle \mathrm{P} 130 / \mathrm{K} 131 \mathrm{~S} / \mathrm{K} 132 \mathrm{~S}$ using LVAX was also observed on xylooligosaccharides with a 2.5 and 2.4-fold increase in catalytic efficiency, respectively using xylopentaose, as compared to wt-PgXynB (Table 4). In the PgXynB model, Lys131 $1_{\text {PgXynB }}$ and Lys132 $2_{\text {PgXynB }}$ form salt bridges with Asp123 $3_{\operatorname{PgX} X n B}$ and Asp128 $8_{\operatorname{PgX} X n B}$, respectively. Asp123 $3_{\operatorname{PgXynB}}$,

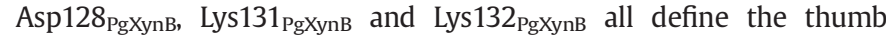
loop and it is expected that the two salt bridges, in the presence of Pro130, maintain the thumb in a position that would impede flexibility as it could lock the loop in a conformation that should hamper oligoxylose binding. In the K131S/K132S and $\Delta \mathrm{P} 130 / \mathrm{K} 131 \mathrm{~S} / \mathrm{K} 132 \mathrm{~S}$ mutants, abolition of the salt bridges by substitution of Lys $131_{\operatorname{PgXynB}}$ and Lys $132_{\text {PgXynB }}$ by the Serine residues could aid the thumb to recover some of the plasticity observed in PgXynA [26].

We further investigated inhibition sensitivity to XIP-I of the mutated xylanases compared to wt-PgXynB. Neither the deletion of Pro130 ( $\triangle \mathrm{P} 130)$ nor the replacement of the two Lysines by Serines (K131S/K132S) altered the inhibition profile of the mutated enzymes a

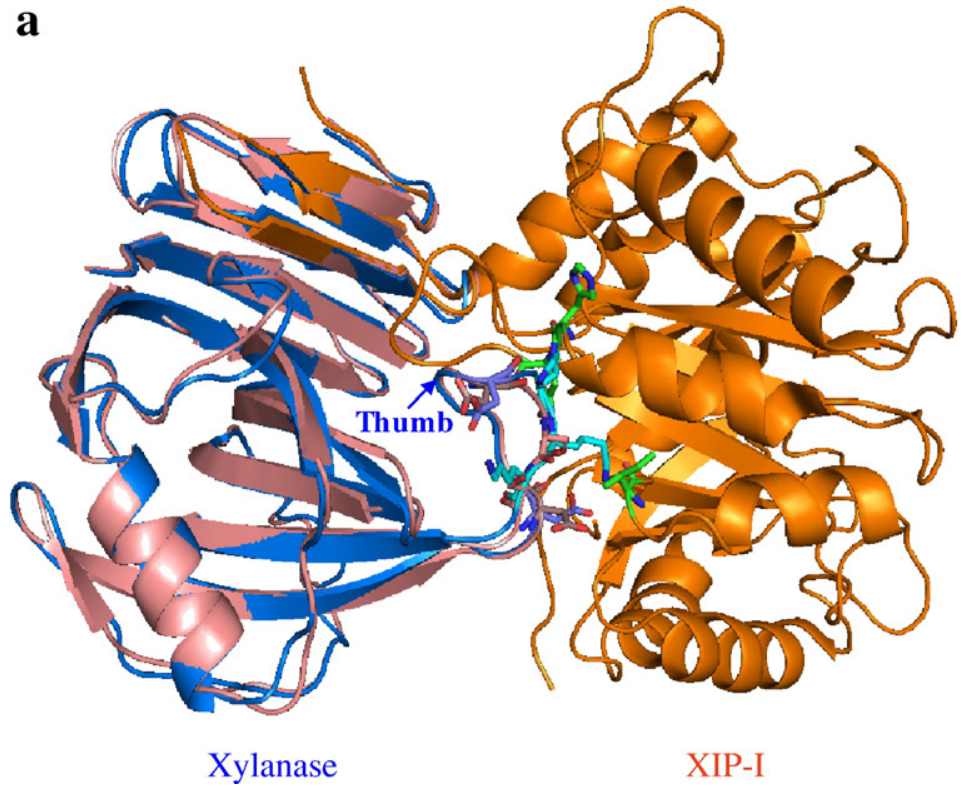

b

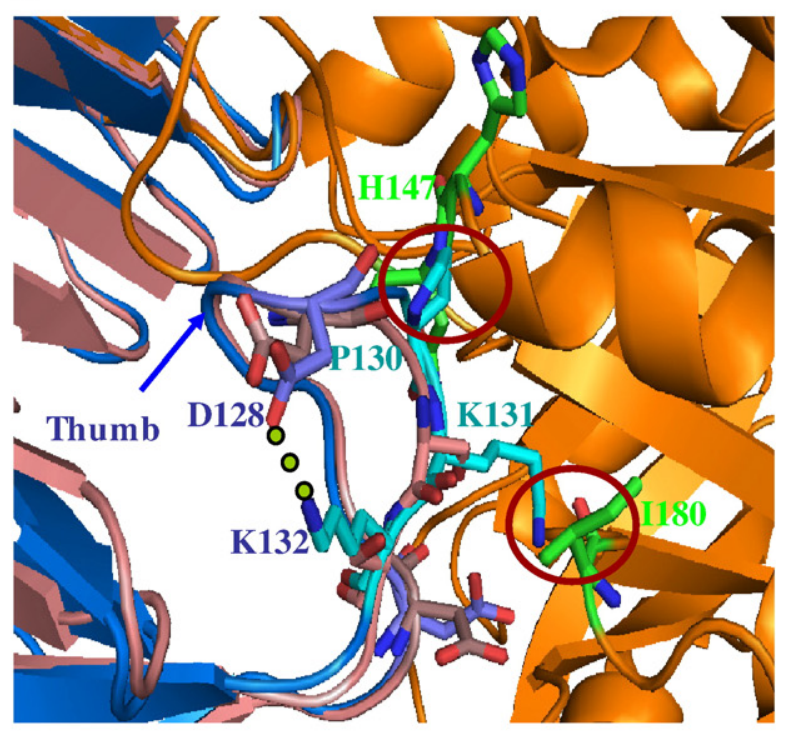

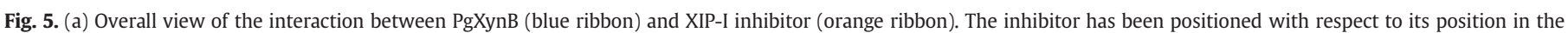

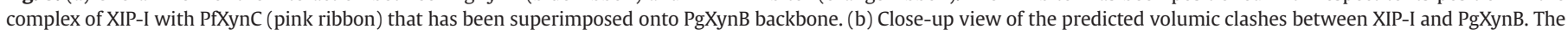

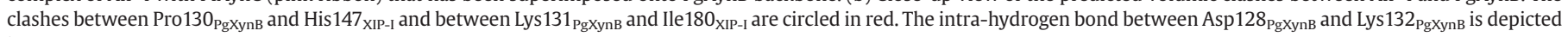
in green. 
whereas the triple mutation $\Delta \mathrm{P} 130 / \mathrm{K} 131 \mathrm{~S} / \mathrm{K} 132 \mathrm{~S}$ conferred inhibition sensitivity to XIP-I with a $K i=16.5 \mathrm{nM}$ (Table 2). The presence of Pro130, Lys131 and Lys132 in wt-PgXynB may hamper the binding of XIP-I, by impeding the flexibility required upon XIP-I binding to the "thumb" region. In the model of XIP-I complexed to PgXynB (see Materials and methods), strong steric clashes are observed between Pro130 $0_{\operatorname{PgXynB}}$ and Asn146 ${ }_{\mathrm{XIP-1}}$; Pro130 13 PgXnB and His $147_{\text {XIP-I }}$; Lys $131_{\text {PgXynB }}$ and Ile $180_{\text {XIP-I }}$ whereas Lys $132_{\text {PgXynB }}$ is involved in H-bond network, enhancing the "thumb" rigidity (Fig. 5) These interactions are not seen in the model of the $\Delta \mathrm{P} 130 / \mathrm{K} 131 \mathrm{~S} /$ K132S mutant, thus corroborating the experimental data. These findings reinforce the important role of the "thumb" region in the resistance of fungal GH11 xylanases to XIP-I. Although insertion of an amino acid in the loop region after the conserved Glycine (Asp130 ${ }_{\text {PgXynA }}$, Pro130 $_{\text {PgXynB }}$ ) is an important criterion of resistance to XIP-I, the deletion of this residue is not enough to restore inhibition sensitivity and it is essential that the loop region around this residue acquires optimum conformation.

In summary, we have investigated the structure-specificity relationships of $P$. griseofulvum xylanases, identifying important residues responsible for substrate and inhibitor specificity of PgXynA and PgXynB.

\section{Acknowledgements}

This study was partly funded by the European Commission in the Communities 6th Framework Programme, Project HEALTHGRAIN (FOOD-CT-2005-514008). This publication reflects only the author's views and the Community is not liable for any use that may be made of the information contained in this publication. We thank Claude Villard for mass spectrometry analysis, Christophe Courtin for providing purified wheat arabinoxylan substrates with different $\mathrm{A} /$ $\mathrm{X}$ ratio, David Giganti for assistance with the manuscript revision and Puratos for support with the publication costs.

\section{References}

[1] L. Saulnier, P.E. Sado, G. Branlard, G. Charmet, F. Guillon, Wheat arabinoxylans: exploiting variation in amount and composition to develop enhanced varieties, J. Cereal Sci. 46 (2007) 261-281.

[2] K.W. Waldron, C.B. Faulds, Cell wall polysaccharides: composition and structure, in: J. Kamerling, G.J. Boons, Y. Lee, A. Suzuki, N. Taniguchi, A.G.J. Voragen (Eds.) Comprehensive Glycoscience: Analysis of Glycans/Polysaccharide Functional Properties, Amsterdam, Elsevier B.V., 2007, pp. 181-201.

[3] N. Kulkarni, A. Shendye, M. Rao, Molecular and biotechnological aspects of xylanases, FEMS Microbiol. Rev. 23 (1999) 411-456.

[4] S. Subramaniyan, P. Prema, Biotechnology of microbial xylanases: enzymology, molecular biology, and application, Crit. Rev. Biotechnol. 22 (2002) 33-64.

[5] T. Collins, C. Gerday, G. Feller, Xylanases, xylanase families and extremophilic xylanases, FEMS Micobiol. 29 (2005) 3-23.

[6] K.K. Wong, L.U. Tan, J.N. Saddler, Multiplicity of beta-1,4-xylanase in microorganisms: functions and applications, Microbiol. Rev. 52 (1988) 305-317.

[7] D.J. Senior, P.R. Mayers, D. Miller, R. Sutcliffe, L. Tan, J.N. Saddler, Selective solubilization of xylan in pulp using a purified xylanase from Trichoderma harzianum, Biotechnol. Lett. 10 (1988) 907-912.

[8] M.L. Polizeli, A.C. Rizzatti, R. Monti, H.F. Terenzi, J.A. Jorge, D.S. Amorim, Xylanases from fungi: properties and industrial applications, Appl. Microbiol. Biotechnol. 67 (2005) 577-591.

[9] J.G. Berrin, N. Juge, Factors affecting xylanase functionality in the degradation of arabinoxylans, Biotechnol. Lett. 30 (2008) 1139-1150.

[10] P.M. Coutinho, B. Henrissat, Carbohydrate-active enzymes: an integrated database approach, in: H.J. Gilbert, G. Davies, B. Henrissat, B. Svensson (Eds.), Recent Advances in Carbohydrate Bioengineering, The Royal Society of Chemistry, Cambridge, 1999, pp. 3-12.

[11] P. Biely, M. Vrsanská, M. Tenkanen, D. Kluepfel, Endo-beta-1,4-xylanase families: differences in catalytic properties, J. Biotechnol. 57 (1997) 151-166.

[12] G.W. Harris, J.A. Jenkins, I. Connerton, N. Cummings, L. Lo Leggio, M. Scott, G.P. Hazlewood, J.I. Laurie, H.J. Gilbert, R.W. Pickersgill, Structure of the catalytic core of the family F xylanase from Pseudomonas fluorescens and identification of the xylopentaose-binding sites, Structure 2 (1994) 1107-1116.

[13] A. Törrönen, J. Rouvinen, Structural and functional properties of low molecular weight endo-1,4-beta-xylanases, J. Biotechnol. 57 (1997) 137-149.

[14] Y. Sato, Y. Niimura, K. Yura, M. Go, Module-intron correlation and intron sliding in family F/10 xylanase genes, Gene 238 (1999) 93-101.
[15] H. Tanaka, T. Nakamura, S. Hayashi, K. Ohta, Purification and properties of an extracellular endo-1,4-beta-xylanase from Penicillium citrinum and characterization of the encoding gene, J. Biosci. Bioeng. 100 (2005) 623-630.

[16] N. Juge, Plant protein inhibitors of cell wall degrading enzymes, Trends Plant. Sci. 11 (2006) 359-367.

[17] R. Chávez, P. Bull, J. Eyzaguirre, The xylanolytic enzyme system from the genus Penicillium, J. Biotechnol. 123 (2006) 413-433.

[18] R. Chávez, F. Fierro, F. Gordillo, J. Francisco Martín, J. Eyzaguirre, Electrophoretic karyotype of the filamentous fungus Penicillium purpurogenum and chromosomal location of several xylanolytic genes, FEMS Microbiol. Lett. 205 (2001) 379-383.

[19] M. Wakiyama, H. Tanaka, K. Yoshihara, S. Hayashi, K. Ohta, Purification and properties of family-10 endo-1,4-beta-xylanase from Penicillium citrinum and structural organization of encoding gene, J. Biosci. Bioeng. 105 (2008) 367-374.

[20] C.S. Furniss, G. Williamson, P.A. Kroon, The substrate specificity and susceptibility to wheat inhibitor proteins of Penicillium funiculosum xylanases from a commercial enzyme preparation, J. Sci. Food Agric. 85 (2005) 574-582.

[21] C.S. Furniss, N.J. Belshaw, M.J. Alcocer, G. Williamson, G.O. Elliott, K. Gebruers, N.P. Haigh, N.M. Fish, P.A. Kroon, A family 11 xylanase from Penicillium funiculosum is strongly inhibited by three wheat xylanase inhibitors, Biochim. Biophys. Acta 1598 (2002) 24-29.

[22] E.X.F. Filho, J. Puls, M.P. Coughlan, Biochemical characteristics of two endo- $\beta$ xylanases produced by Penicillium capsulatum, J. Ind. Microbiol. 11 (1993) 171-180.

[23] S.E. Ryan, K. Nolan, R. Thompson, G.M. Gubitz, A.V. Savage, M.G. Tuohy, Purification and characterization of a new low molecular weight endoxylanase from Penicillium capsulatum, Enzyme Microb. Technol. 33 (2003) 775-785.

[24] J.L. Jonniaux, D. Bruyer, F. Arnaut, T. Dauvrin, A new xylanase from Penicillium griseofulvum, Meded. Rijksuniv. Gent Fak. Landbouwkd. Toegep. Biol. Wet. 66 (2001) 261-266

[25] J.G. Berrin, E.H. Ajandouz, J. Georis, F. Arnaut, N. Juge, Substrate and product hydrolysis specificity in family 11 glycoside hydrolases: an analysis of Penicillium funiculosum and Penicillium griseofulvum xylanases, Appl. Microbiol. Biotechnol. 74 (2007) 1001-1010.

[26] G. André-Leroux, J.G. Berrin, J. Georis, F. Arnaut, N. Juge, Structure-based mutagenesis of Penicillium griseofulvum xylanase using computational design, Proteins 72 (2008) 1307-1898.

[27] J.L. Jonniaux, T. Dauvrin, Enzyme with xylanase activity, EP Patent (2001) 1,130,102-A1.

[28] S.N. Ho, H.D. Hunt, R.M. Horton, J.K. Pullen, L.R. Pease, Site-directed mutagenesis by overlap extension using the polymerase chain reaction, Gene 77 (1989) 51-59.

[29] M.J. Bailey, P. Biely, K. Poutanen, Interlaboratory testing of methods for assay of xylanase activity, J. Biotechnol. 23 (1992) 257-270.

[30] W.R. McLauchlan, M.T. Garcia-Conesa, G. Williamson, M. Roza, P. Ravestein, J. Maat, A novel class of protein from wheat which inhibits xylanases, Biochem. J. 338 (1999) 441-446.

[31] I. Matsui, K. Ishikawa, E. Matsui, S. Miyairi, S. Fukui, K. Honda, Subsite structure of Saccharomycopsis alpha-amylase secreted from Saccharomyces cerevisiae, J. Biochem. (Tokyo) 109 (1991) 566-569.

[32] F. Payan, P. Leone, S. Porciero, C. Furniss, T. Tahir, G. Williamson, A. Durand, P. Manzanares, H.J. Gilbert, N. Juge, A. Roussel, The dual nature of the wheat xylanase protein inhibitor XIP-I: structural basis for the inhibition of family 10 and family 11 xylanases, J. Biol. Chem. 279 (2004) 36029-36037.

[33] M.A. Larkin, G. Blackshields, N.P. Brown, R. Chenna, P.A. McGettigan, H. McWilliam, F. Valentin, I.M. Wallace, A. Wilm, R. Lopez, J.D. Thompson, T.J. Gibson, D.G. Higgins, ClustalW and ClustalX version 2, Bioinformatics 23 (2007) 2947-2948.

[34] A. Salí, T.L. Blundell, Comparative protein modelling by satisfaction of spatial restraints, J. Mol. Biol. 234 (1993) 779-815

[35] R.A. Laskowski, M.W. MacArthur, D.S. Moss, J.M. Thornton, PROCHECK: a program to check the stereochemical quality of protein structures, J. Appl. Cryst. 26 (1993) 283-291.

[36] E. Sabini, K.S. Wilson, S. Danielsen, M. Schulein, G.J. Davies, Oligosaccharide binding to family 11 xylanases: both covalent intermediate and mutant product complexes display $(2,5)$ B conformations at the active center, Acta Crystallogr. D Biol. Crystallogr. 57 (2001) 1344-1347.

[37] M. Vardakou, C. Dumon, J.W. Murray, P. Christakopoulos, D.P. Weiner, N. Juge, R.J. Lewis, H.J. Gilbert, J.E. Flint, Understanding the structural basis for substrate and inhibitor recognition in eukaryotic GH11 xylanases, J. Mol. Biol. 375 (2008) 1293-1305.

[38] J.E. Lennard-Jones, Cohesion, Proc. Physic. Soc. 43 (1931) 461-482.

[39] T. Beliën, S. Van Campenhout, M. Van Acker, J. Robben, C.M. Courtin, J.A. Delcour, G. Volckaert, Mutational analysis of endoxylanases XylA and XylB from the phytopathogen Fusarium graminearum reveals comprehensive insights into their inhibitor insensitivity, Appl. Environ. Microbiol. 73 (2007) 4602-4608.

[40] T. Beliën, S. Van Campenhout, A. Vanden Bosch, T.M. Bourgois, S. Rombouts, J. Robben, C.M. Courtin, J.A. Delcour, G. Volckaert, Engineering molecular recognition of endoxylanase enzymes and their inhibitors through phage display, J. Mol. Recognit. 20 (2007) 103-112.

[41] G. Paës, V. Tran, M. Takahashi, I. Boukari, M.J. O'Donohue, New insights into the role of the thumb-like loop in GH-11 xylanases, Protein Eng. Des. Sel. 20 (2007) $15-23$.

[42] R. Flatman, W.R. McLauchlan, N. Juge, C.S. Furniss, J.G. Berrin, R.K. Hughes, P. Manzanares, J.E. Ladbury, R. O'Brien, G. Williamson, Interactions defining the specificity between fungal xylanases and the xylanase-inhibiting protein XIP-I from wheat, Biochem. J. 365 (2002) 773-781.

[43] P. Gouet, E. Courcelle, D.I. Stuart, F. Metoz, ESPript: multiple sequence alignments in PostScript, Bioinformatics 15 (1999) 305-308. 